\title{
Comparison of Particle Swarm Optimization and Ziegler Nichols Techniques
}

\author{
${ }^{1}$ Dipali Joshi-Jain, ${ }^{2}$ Gaurav S. Gunjal, ${ }^{3}$ Sahil S. Hadkar, ${ }^{4}$ Prasad S. Deshmukh, ${ }^{5}$ Siddhant V. Sanap \\ ${ }^{1}$ Assistant Professor, ${ }^{2,3,4,5}$ Student, RGIT, Mumbai-India. \\ 1dipaishaj@gmail.com, ${ }^{2}$ gauravgunjal98@gmail.com, ${ }^{3}$ sahilhadkar17@gmail.com, \\ 4deshmukhprasad211@gmail.com, 5siddhant.sanap98@gmail.com
}

Abstract- Two tuning techniques namely: Particle Swarm Optimization (PSO) and Ziegler Nichols (ZN) technique are compared. PSO is an optimization technique based on the movement and intelligence of swarms. PSO applies the concept of social interaction to problem solving. It is a computational method that optimizes a problem by iteratively trying to improve a candidate solution about a given measure of quality. Ziegler Nichols tuning method is a heuristic method of tuning a PID controller. The ZN close loop tuning is performed by setting the I (integral) and D (derivative) gains to zero and increasing proportional gain to obtain sustained oscillations. The DC Motor is represented by second order transfer function is used as a plant, which is controlled using PID controller. The PID controller parameters are chosen by tuning the controller using PSO algorithm and ZN method. The response of the system to unit step input is plotted and performance measures are evaluated for comparing PSO algorithm and ZN technique. Here we have compared the two tuning methods based upon the settling time (Ts), peak overshoot (Mp) and the two performance indices namely Integral square error (ISE) and Integral Absolute error (IAE).

Keywords- Integral Absolute error (IAE), Integral square error (ISE), Peak overshoot (Mp), Particle Swarm Optimization (PSO), settling time (Ts), Ziegler Nichols (ZN) .

\section{INTRODUCTION}

PSO, [1] that belongs to a class of Meta-Heuristic Algorithm, is an Intelligent Optimization Algorithm. It is inspired by the social behavior of birds and animals like fishes. It is a powerful algorithm and has applications in various fields of science like machine learning, data mining, operational research, etc. Initially, it was introduced by James Kennedy and Russel C Eberhart in 1995. PSO is a Swarm Intelligence Method and it uses some intelligent agents called "particles" to reach another level of intelligence that is unreachable for any particle in a swarm. However, the performance of PSO still has space for improvement. The main algorithm of PSO is simple. It has a straightforward implementation. It is easy to perform as there are few parameters which we need to adjust. PSO offers the bigger optimization ability when compared with the other developing calculations and it can be completed easily. During the development of several generations, only the most optimist particle can transmit information onto the particles and the speed of the research is very fast.

\section{METHOD}

The proposed system used for comparing the performance of PSO and ZN tuning method is as shown in figure 1 . The D.C. Motor represented with following second order transfer function:-

$$
\text { T. F }=\frac{0.01}{0.005 s^{2}+0.06 s+0.1001}
$$

is used as a plant which is controlled using PID controller. The tuning parameters of PID controller are set by using PSO algorithm and then Z-N method. The system is evaluated for its performance by comparing different performance measures like settling time (Ts), peak overshoot (Mp) and the two performance indices namely Integral square error (ISE) and Integral Absolute error (IAE).

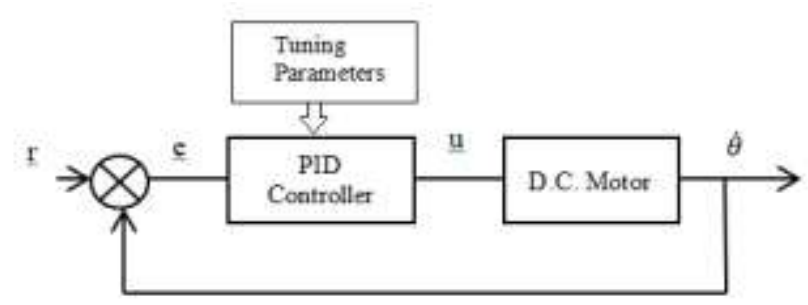

Figure 1: D.C. Motor control using PID controller 


\section{A. Mathematical model of PSO:}

The position and velocity of every particle is updated by using vector representation of PSO as shown in figure 2. The particle moves towards the new position $\mathrm{V}_{\mathrm{i}}(\mathrm{t}+1)$, all the 3 vector components shown in the figure 2 helps us find the newly updated position [2]. The new position here is created according to previous velocity, the personal and global best which is probably a better location based on the previous experience $V_{i}(t+1)$.

$\mathrm{P}_{\mathrm{i}}(\mathrm{t})=$ personal best solution

$\mathrm{g}(\mathrm{t})=\mathrm{global}$ best /common best solution

$\mathrm{x}_{\mathrm{i}}(\mathrm{t}+1)=$ better $/$ optimal solution using PSO.

$\mathrm{V}_{\mathrm{i}}(\mathrm{t}+1)=$ new velocity obtained by addition of 3 vectors

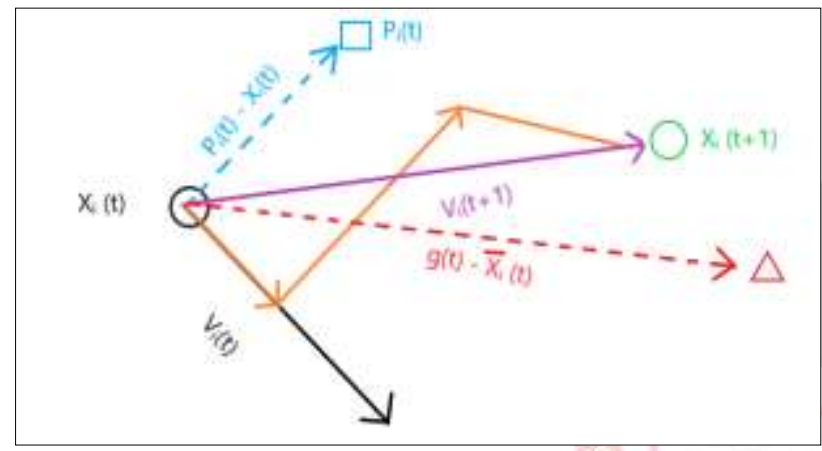

Figure 2: Vector Representation of PSO

B. The Existing PSO Algorithm

Figure 3 show the general flowchart of the PSO algorithm. The main steps in the particle swarm optimization process are described as follows [3]:

Input: Initialization of PID controller parameters $(K p, K$, $K d$, Population size, $C 1, C 2$ and $\omega)$

Output: Optimal PID control parameters and transient response $(K p, K, K d, t s, t r, M p, I S E)$

\section{BEGIN Algorithm}

Step1: Setting PSO parameters - Set the population size NP, Cognitive Component $C 1$, Social Component $C 2$ and inertia weight $\omega$. The problem dimension is set based on number of parameters used in the objective function. In this case, problem dimension refers to the number of PID parameters $K p, K, K d$ which is equal to 3 .

Step 2: Initialize the vector population - Initialize all vector population randomly in the given upper and lower bound

$\operatorname{Pop}_{\mathrm{ij}}=\mathrm{L}+(\mathrm{H}-\mathrm{L})$.

$\operatorname{rand}_{\mathrm{ij}}(0,1)=$ random number between 0 and 1 ;

for $\mathrm{i}=1 \ldots \ldots, \mathrm{D}, \mathrm{j}=1 \ldots \ldots . \mathrm{NP}$

Step 3: Evaluate the fitness of each vector.

$F_{i t}=f\left(P o p_{j}\right)$
Where $f\left(\left(\right.\right.$ Pop $\left._{j}\right)=\int_{0}^{\tau}(e(t))^{2} d t$, for $I S E$

Before optimization starts, the population needs to be initialized. And their fitness values need to be evaluated. The population is initialized randomly within its boundary constraints. The fitness value which referred as ISE is computed based on error of control system.

Step 4: Compare the individual fitness of each particle to its previous $p_{\text {best }}$. Thus the optimum fitness can be obtained by updating the fitness with the new $p_{\text {best }}$.

Step 5: Compare evaluation value of each individual with its $p_{\text {best }}$. The best evaluation value among the $p_{\text {best }}$ is denoted as $g_{\text {best }}$.

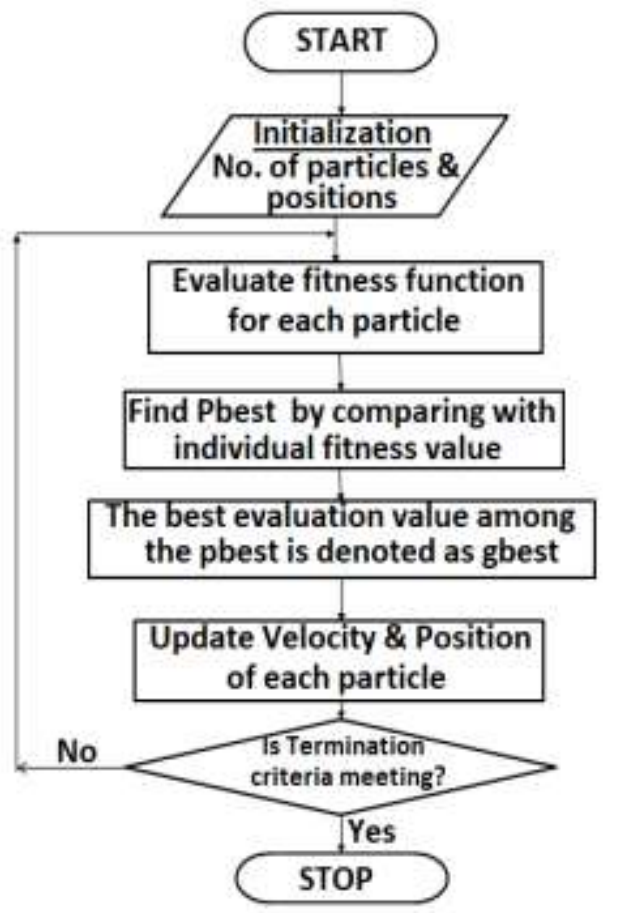

Figure 3: PSO Algorithm Chart

Step 6: Update the velocity, position, $g_{\text {best }}$, $p_{\text {best }}$ of particles.

Step 7: If the number of iterations reaches the maximum then, go to Step 8. Otherwise, go to Step 2.

Step 8: The individual that generates the latest $g_{\text {best }}$ is an optimal controller parameter.

\section{END Algorithm}

The PSO algorithm is developed by writing a code using Matlab software. The algorithm is initialized by setting number of particles and their positions.

- Number of particles: The number of particles is typically in the range [20, 40].

- Position of particles: The random positions are assigned to the particles using random vector.

- Inertia Weight: The inertia weight is around 0.4 to 0.9 , and as the algorithm progresses this value is gradually decreased to 0 . 
- The upper and lower bound on the values of $\mathrm{K}_{\mathrm{p}}$, $\mathrm{K}_{\mathrm{i}}$ and $\mathrm{K}_{\mathrm{d}}$ are set as [250 0 ], [60 0 ] and [250 0 ] respectively.

After initializing the particles, fitness function for each particle is evaluated with the fitness function of D.C. motor. By using this fitness function, fitness value i.e. ISE is computed. The best position of each individual particle is calculated by comparing its value with the previous value which provides $p_{\text {best }}$ value. The value of $p_{\text {best }}$ that fits close to the fitness function is considered as $g_{\text {best }}$. In the proposed system, the controller parameter which provides optimal value of $g_{\text {best }}$ is obtained in 150 iterations.

The process is terminated as the error between the objective function value and the best fitness value is less than a threshold.

The controller parameters are then set and the system response is plotted for unit step input using Matlab software.

\section{Ziegler-Nichols Tuning Method}

The tuning the PID controller is then carried out using Z-N method and performance of the system shown in figure 1, is then evaluated. Ziegler Nichols tuning method [4] is most commonly used to tune a PID controller. It was developed by John G. Ziegler and Nathaniel Nichols. The Ziegler Nichols tuning method was introduced in 1940 and made a huge impact in making PID feedback controls acceptable to control engineers [4]. The tuning of the PID controller using Ziegler Nichols technique can be done by two methods namely:

\section{Ziegler Nichols open-loop tuning method}

2. Ziegler Nichols closed-loop tuning method

The proposed system uses close loop tuning method for determining the values of $\mathrm{K}_{\mathrm{p}}, \mathrm{T}_{\mathrm{i}}$ and $\mathrm{T}_{\mathrm{d}}$. Following are the rules related to Closed-loop tuning method:

Step 1- If the process is rapidly oscillating; bring the process to normal condition.

Step 2- Eliminate integral and derivative control action initially by setting $\mathrm{T}_{\mathrm{i}}=\infty$ and $\mathrm{T}_{\mathrm{d}}=0$.

Step 3- Introduce proportional control $\left(\mathrm{K}_{\mathrm{p}}\right)$ in the process

Step 4- Keep on increasing the value of $K_{p}$ until sustained oscillations are obtained.

Step 5- Find out the value of Ultimate Gain $\left(\mathrm{K}_{\mathrm{u}}=\mathrm{K}_{\mathrm{cr}}\right)$ and ultimate period $\left(\mathrm{P}_{\mathrm{u}=} \mathrm{P}_{\mathrm{cr}}\right)$ at which the oscillation has begun into the system.

Step 6- Calculate various parameters $\left(\mathrm{K}_{\mathrm{c}}, \mathrm{T}_{\mathrm{i}}, \mathrm{T}_{\mathrm{d}}\right)$ from the table given below.

The new settings for the controller i.e. the values $K_{p}, T_{i}$ and $T_{d}$. are calculated from the equations given in the table below. The unit step input is then applied to the system and the response is plotted using Matlab.

Table 1:Ziegler Nichols Tuning parameters

\begin{tabular}{|c|c|c|c|}
\hline $\begin{array}{c}\text { Type of } \\
\text { Controller }\end{array}$ & $\mathbf{K}_{\mathbf{p}}$ & $\mathbf{T}_{\mathbf{i}}$ & $\mathbf{T}_{\mathbf{d}}$ \\
\hline $\mathbf{P}$ & $0.5 \mathrm{~K}_{\mathrm{cr}}$ & $\infty$ & 0 \\
\hline $\mathbf{P I}$ & $0.45 \mathrm{~K}_{\mathrm{cr}}$ & $\frac{\mathbf{1}}{\mathbf{1 . 2}} \boldsymbol{P}_{c r}$ & 0 \\
\hline PID & $0.6 \mathrm{~K}_{\mathrm{cr}}$ & $0.5 \mathrm{P}_{\mathrm{cr}}$ & $0.125 \mathrm{P}_{\mathrm{cr}}$ \\
\hline
\end{tabular}

The Ziegler-Nichols method is quicker and easier to use than other methods. It is a robust and popular method.

\section{RESUlTS}

The comparison between the two tuning methods, PSO and $\mathrm{Z}-\mathrm{N}$ is performed based upon the settling time $\left(\mathrm{T}_{\mathrm{s}}\right)$, Peak overshoot $\left(\mathrm{M}_{\mathrm{p}}\right)$ and the two performance indices namely Integral square error (ISE) and Integral Absolute error (IAE). The DC Motor is considered as a plant, which is controlled using PID controller [5]. The PID controller parameters are tuned using PSO and $\mathrm{ZN}$ tuning methods for the above system transfer function. The response of the system for unit step input is as shown in figure 4 (a) and figure 4 (b). Figure 4 (a) shows the response when the PID controller is tuned by using the parameters obtained from PSO algorithm and figure 4 (b) shows the response when the PID controller is tuned by using the parameters of the $\mathrm{ZN}$ method.

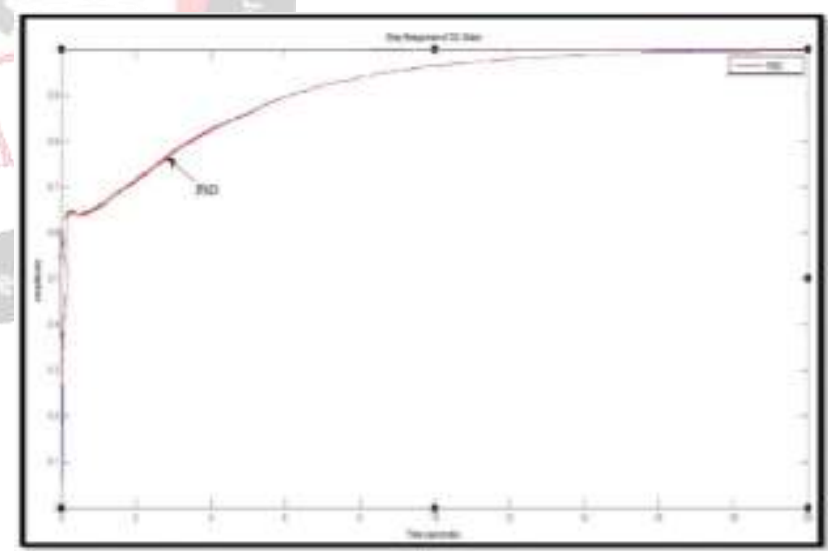

Figure 4 (a): PSO Tuning Method

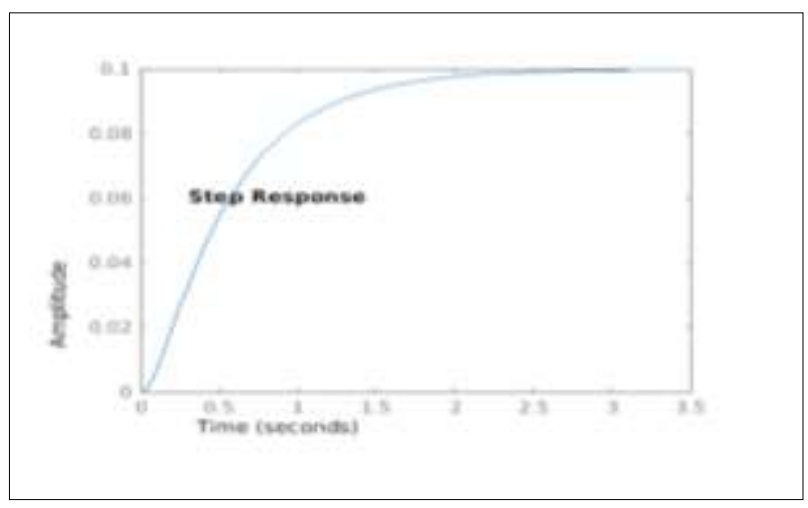

Figure 4 (b): Ziegler Nichols Tuning Method 
The table 2 shows the values of $\mathrm{K}_{\mathrm{p}}, \mathrm{K}_{\mathrm{i}}, \mathrm{K}_{\mathrm{d}}$ obtained from PSO and ZN method. The value of $\mathrm{K}_{\mathrm{p}}=6.06, \mathrm{~K}_{\mathrm{i}}=2.016$ and $\mathrm{K}_{\mathrm{d}}=0.124$, when PID controller is tuned by using Ziegler-Nichols method whereas the value of $\mathrm{K}_{\mathrm{p}}=15.0880$, $\mathrm{K}_{\mathrm{i}}=5.5075$ and $\mathrm{K}_{\mathrm{d}}=10$, when PID controller is tuned by using PSO algorithm. It has been observed that there is significant improvement in the parameter values when the controller is tuned using PSO algorithm. The value of $\mathrm{Kp}$ is improved by $59.83 \%, \mathrm{~K}_{\mathrm{i}}$ by $63.39 \%$ and $\mathrm{K}_{\mathrm{d}}$ by 98.76\%. The performance measures $\mathrm{T}_{\mathrm{s}}$ and $\mathrm{M}_{\mathrm{p}}$ shows that system tuned using Z-N method, comparatively has less settling time $\left(\mathrm{T}_{\mathrm{s}}\right)$ than the system tuned using PSO and thus respond faster while the peak overshoot $\left(\mathrm{M}_{\mathrm{p}}\right)$ for both the system is zero and the response is critically damped as shown in figure 4 (a) and figure 4 (b). The two performance indices, Integral square error (ISE) and Integral Absolute error (IAE) shows that the system tuned using PSO algorithm gives better performance by improving the values of ISE and IAE with $15.27 \%$ and $9.09 \%$ improvement in the system performance [6].

Table 2:Performance Indices for PSO and ZN tuning methods

\begin{tabular}{|c|c|c|c|}
\hline PARAMETER & $\begin{array}{l}\text { PSO } \\
\text { ALGORITHM }\end{array}$ & $\begin{array}{l}\text { ZIEGLER } \\
\text { NICHOLS } \\
\text { TUNING }\end{array}$ & \%IMPROVMENT \\
\hline $\mathbf{K p}$ & $15.0880^{*}$ & 6.06 & $59.83 \%$ \\
\hline $\mathbf{K i}$ & $5.5075^{*}$ & 2.016 & $63.39 \%$ \\
\hline $\mathbf{K d}$ & $10^{*}$ & 0.124 & $98.76 \%$ \\
\hline $\mathbf{T}_{\mathbf{s}}(\mathbf{s e c})$ & 12.2616 & 2.07 & $83.11 \%$ \\
\hline $\mathbf{M}_{\mathbf{p}}$ & 0 & 0 & $0 \%$ \\
\hline $\mathbf{I S E}$ & $0.004^{*}$ & 0.004717 & $15.27 \%$ \\
\hline IAE & $0.02^{*}$ & 0.022 & $9.09 \%$ \\
\hline
\end{tabular}

NOTE - ' $*$ ' is represented as improvement in parameter.

This limitation occurs as each iteration in PSO takes certain amount of time, so the overall time increases. Hence, the existing PSO algorithm does have certain shortcomings like the number of iterations, considerable execution time and error. To overcome these limitations, a modification to the existing PSO algorithm to improve its performance in terms of number of iterations, the execution time and the transient response, it is suggested to combine the mutation operators [7] like Cauchy [8,9], Levy and Gaussian along with the existing PSO algorithm. This helps in improving the performance as compared to the existing algorithm. The figure 5 shows the step response of the system, when PSO algorithm is combined with Levy, Cauchy and Gaussian mutation operators. The response shows improvement in the transient parameters like $\mathrm{Ts}$, $\mathrm{Tr}$, i.e. reduction in setting time, rise time.

The response of PID controller tuned with the existing PSO algorithm, for DC Motor, as shown in figure, shows the transient parameters like Settling time $\left(\mathrm{T}_{\mathrm{s}}\right)$, the rise time $\left(\mathrm{T}_{\mathrm{r}}\right)$, and Peak Overshoot $\left(\mathrm{M}_{\mathrm{p}}\right)$ are more. The output of the proposed PSO algorithm with mutation operator, for DC Motor, shows improvement in the transient parameters like $T_{s}, T_{r}, M_{p}$ i.e. reduction in the Settling time $\left(T_{s}\right)$, the rise time $\left(T_{r}\right)$, and Peak Overshoot $\left(M_{p}\right)$ shown in figure 5 as compared to the existing PSO algorithm.

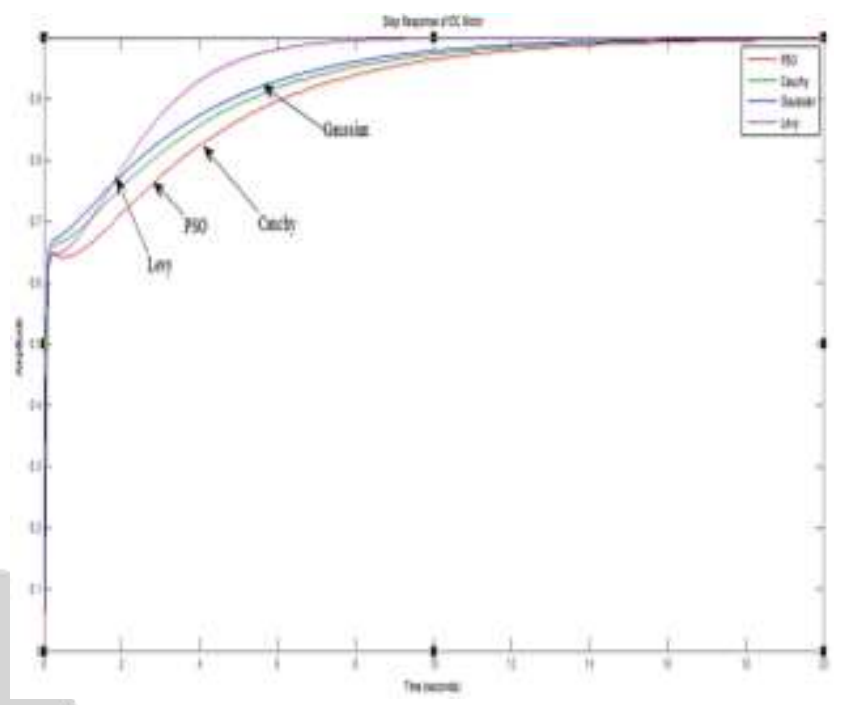

Figure 5: Step response of D.C. Motor when PID controller is tuned by using PSO algorithm combined with mutation operators.

\section{Conclusion}

In this paper, we have tried to prove that the performance of PID controller tuning based on the PSO algorithm is better than the Ziegler Nichols tuning technique. The D.C. motor with the transfer function

$$
\text { T. F }=\frac{0.01}{0.005 s^{2}+0.06 s+0.1001}
$$

is considered as a plant. The PID controller is used to control the plant and tuning of controller is done using PSO algorithm and Z-N method. For the comparison of the performance, we have considered four performance indices namely settling time $\left(\mathrm{T}_{\mathrm{s}}\right)$, peak overshoot $\left(\mathrm{M}_{\mathrm{p}}\right)$, Integral Square Error (ISE) and Integral Absolute Error (IAE). As shown in the table 2 for DC motor system, it depicts that the Integral Square Error (ISE) and Integral Absolute Error (IAE) value tends to decrease by using the proposed PSO algorithm as compared to the Ziegler Nichols method for PID tuning. From the comparison of the above-mentioned performance indices we have observed that there is:

\section{1. $15.27 \%$ improvement in ISE}

\section{2. $9.09 \%$ improvement in IAE}

The settling time for the system tuned by PSO algorithm is increased compared to the system tuned using Z-N method as each iteration of PSO takes significant time. While peak overshoot for the system is 0 . Based on the above we can successfully conclude that the PSO algorithm can be suggested as a better tuning technique as compared to the 
Ziegler Nicholas technique in terms of Integral Square Error (ISE) and Integral Absolute Error (IAE).

The performance of PSO algorithm in terms of performance indices like settling time $\left(\mathrm{T}_{\mathrm{s}}\right)$ and rise time $\left(\mathrm{T}_{\mathrm{r}}\right)$ can be improved by using mutation operators combined with PSO algorithm. The table 3 shows the performance measures using PSO algorithm and PSO algorithm combine with the Cauchy mutation operator.

Table 3: Performance Indices for PSO combined with Cauchy mutation operator.

\begin{tabular}{|c|c|c|c|}
\hline PARAMETERS & PSO & CAUCHY & $\begin{array}{l}\text { \% } \\
\text { IMPROVEMENT }\end{array}$ \\
\hline Kp & 15.0880 & 18.1454 & - \\
\hline Ki & 5.5075 & 6.5423 & - \\
\hline Kd & 10 & 10 & - \\
\hline Ts & 12.2616 & 11.1856 & $8.78 \%$ \\
\hline Tr & 6.1305 & 5.256 & $14.26 \%$ \\
\hline Mp & $0 \%$ & $0 \%$ & $0 \%$ \\
\hline ISE & 0.4717 & 0.2106 & $55.35 \%$ \\
\hline
\end{tabular}

The ISE value can be reduced to $55.34 \%$ and the execution time can be reduced by $6.2 \%$ by using the Cauchy Operator. Also, the rise time can be improved by $44.35 \%$ and the Settling time can be improved by $51.28 \%$ due to Levy Operator. Further the number of iterations can also be reduced using the above mutation operators. Thus the PSO algorithm is better than the Ziegler Nichols technique and minimizes the error to improve the performance of the system.

\section{REFERENCES}

[1] J. Kennedy ; R. Eberhart "Particle swarm optimization" IEEE, Proceedings of ICNN'95 - International Conference on Neural Networks, ISBN : 0-7803-2768-3, : 10.1109/ICNN.1995.488968, Perth, WA, Australia, Australia

[2] Iraj Koohi ; Voicu Z. Groza; "Optimizing Particle Swarm Optimization algorithm” 2014 IEEE 27th Canadian Conference on Electrical and Computer Engineering (CCECE), 10.1109/CCECE.2014.6901057, Toronto, ON, Canada

[3] Frans van den Bergh, "An Analysis of Particle Swarm Optimizers," Ph.D. dissertation, Univ. of Pretoria, 2001.

[4] “Zigler Nicholas,” Ziegler-ichols_closed-loop_tuning_method ,2014 ed.

[5] P. M. Meshram ; Rohit G. Kanojiya, "Tuning of PID controller using Ziegler-Nichols method for speed control of DC motor", IEEE, INSPEC Accession Number: 12818603, Nagapattinam, Tamil Nadu, India.

[6] B. Nagaraj; N. Murugananth, "A comparative study of PID controller tuning using GA, EP, PSO and ACO", 2010
International Conference On Communication Control And Computing Technologies, IEEE, 10.1109/ICCCCT.2010.5670571, Ramanathapuram, India

[7] Andrews S.Paul, "An investigation into mutation operators for particle swarm optimization," IEEE Press on Evolutionary Computation, pp. 1044-1051, 2006.

[8] Zhongzhou Du ; Si Li ; Yi Sun ; Nana Li;" Adaptive particle swarm optimization algorithm based on levy flights mechanism" 2017 Chinese Automation Congress (CAC), IEEE, 10.1109/CAC.2017.8242815, Jinan, China

[9] Hui Wang ; Changhe Li ; Yong Liu ; Sanyou Zeng; "A Hybrid Particle Swarm Algorithm with Cauchy Mutation', IEEE”, ISBN: 1-4244-0708-7, 10.1109/SIS.2007.367959, Honolulu, HI, USA

[10] "Particle Swarm Optimization,” Scholarpedia, Oct.15, 2010. 